\title{
Lesson of the month 2: When steroids stop working - infective endocarditis, the great mimicker
}

\author{
Authors: Alberto San Francisco, ${ }^{A}$ James S Tomlinson, ${ }^{B}$ Samuel Walters, ${ }^{\mathrm{C}}$ Sally Curtis ${ }^{\mathrm{D}}$ and Rachael James ${ }^{\mathrm{E}}$
}

\begin{abstract}
A 63-year-old man with an extensive travel history to South East Asia presented with generalised malaise, temporal headaches and high inflammatory markers. He was treated with corticosteroids for presumed giant cell arteritis. Unsuccessful attempts to wean him from prednisolone prompted further investigations by rheumatology, haematology and finally ophthalmology. Roth spots were identified which prompted blood cultures to be taken. All three sets grew Streptococcus sinensis, an alpha-haemolytic Streptococcus reported as an emerging cause of endocarditis worldwide. The patient had signs of severe aortic regurgitation, confirmed on transthoracic echo. A transoesophageal echo demonstrated large aortic valve vegetations. He underwent an aortic valve replacement and completed 6 weeks of intravenous antibiotics with resolution of his symptoms.

This case illustrates the importance of challenging a previous diagnosis, including repeat examination, when a patient's condition does not evolve as expected. Endocarditis is recognised as a great imitator and the diagnosis remains challenging.
\end{abstract}

KEYWORDS: Chronic infective endocarditis, Streptococcus sinensis, emerging infections

\section{Case description}

A 63-year-old Caucasian man presented to the emergency department (ED) with a 3-week history of general malaise, fatigue and gradually worsening pressure-like, temporal headaches. He reported intermittent double vision but denied any other visual or ocular changes, jaw or tongue claudication, fever or night sweats. He owned a silk factory in rural Vietnam and was a frequent traveller to South East Asia. The patient denied any significant past medical history but had undergone dental bridge work in Hong Kong. His inflammatory markers were raised, with an erythrocyte sedimentation rate $69 \mathrm{~mm} / \mathrm{h}$ and a C-reactive protein (CRP) $69.0 \mathrm{mg} / \mathrm{L}$. A presumptive diagnosis of giant cell arteritis

Authors: ${ }^{\mathrm{A}}$ specialist registrar in infectious diseases and microbiology, Royal Sussex County Hospital, Brighton, UK; ${ }^{B}$ core medical trainee, Royal Sussex County Hospital, Brighton, UK; ${ }^{C}$ foundation trainee, Royal Sussex County Hospital, Brighton, UK; ${ }^{\mathrm{D}}$ consultant microbiologist, Royal Sussex County Hospital, Brighton, UK;

${ }^{E}$ consultant cardiologist, Royal Sussex County Hospital, Brighton, UK
(GCA) was made and he was commenced on oral prednisolone 40 mg daily.

The patient was reviewed 2 months later in a rheumatology clinic. He reported complete resolution of symptoms, apart from hot flushes, within 24 hours of starting steroids. He appeared well. Cardiovascular, musculoskeletal and visual field examination was normal. An autoimmune screen (anti-nuclear antibodies, antineutrophil cytoplasmic antibodies), serum plasma electrophoresis and malaria blood films were negative. A temporal artery biopsy was not performed as his symptoms had resolved and steroid treatment would decrease the diagnostic yield. He was instructed to reduce his daily prednisolone dose by $2.5 \mathrm{mg}$ every three weeks. The patient was referred to ophthalmology and had normal fundoscopy and slit-lamp examination.

Two months later, while travelling to Vietnam on business, he inadvertently stopped taking his steroids for a week. He experienced a recurrence of his symptoms and on returning to the UK, presented to the ED, where his prednisolone was increased to $40 \mathrm{mg}$. He was subsequently reviewed multiple times in the rheumatology clinic and attempts to wean his steroid dose were unsuccessful. He underwent positron emission tomography computed tomography to exclude systemic vascular inflammation; diffuse increased bone marrow uptake and hepato-splenomegaly was demonstrated but no evidence of systemic vasculitis (Fig 1). The diagnosis of GCA was challenged and the patient was referred to haematology for further investigation to exclude a lymphoproliferative disease. A blood film showed neutrophilia with toxic granulation (concordant with long-term steroid use) and bone marrow biopsy was normal. The patient was re-referred to ophthalmology to exclude anterior optic neuropathy. Roth spots were visualised (Fig 2).

At this stage, 9 months after his original presentation, his symptoms had progressed, and the patient reported night sweats and fevers. In light of his raised inflammatory markers, foreign travel history and new ophthalmological findings, endocarditis (IE) was considered as an alternative diagnosis and three sets of blood cultures were taken. CRP was $64 \mathrm{mg} / \mathrm{L}$ (Fig 3). All three blood culture sets yielded Streptococcus sinensis. A long diastolic murmur of severe aortic regurgitation was heard, and he was admitted. Intravenous amoxicillin and gentamicin were started. Transthoracic echo confirmed severe aortic regurgitation with several large vegetations on a tricuspid aortic valve. The aortic root appeared normal and PR interval on ECG was normal. A transoesophageal echo confirmed multiple, large vegetations and no evidence of root endocarditis (Fig 4). 


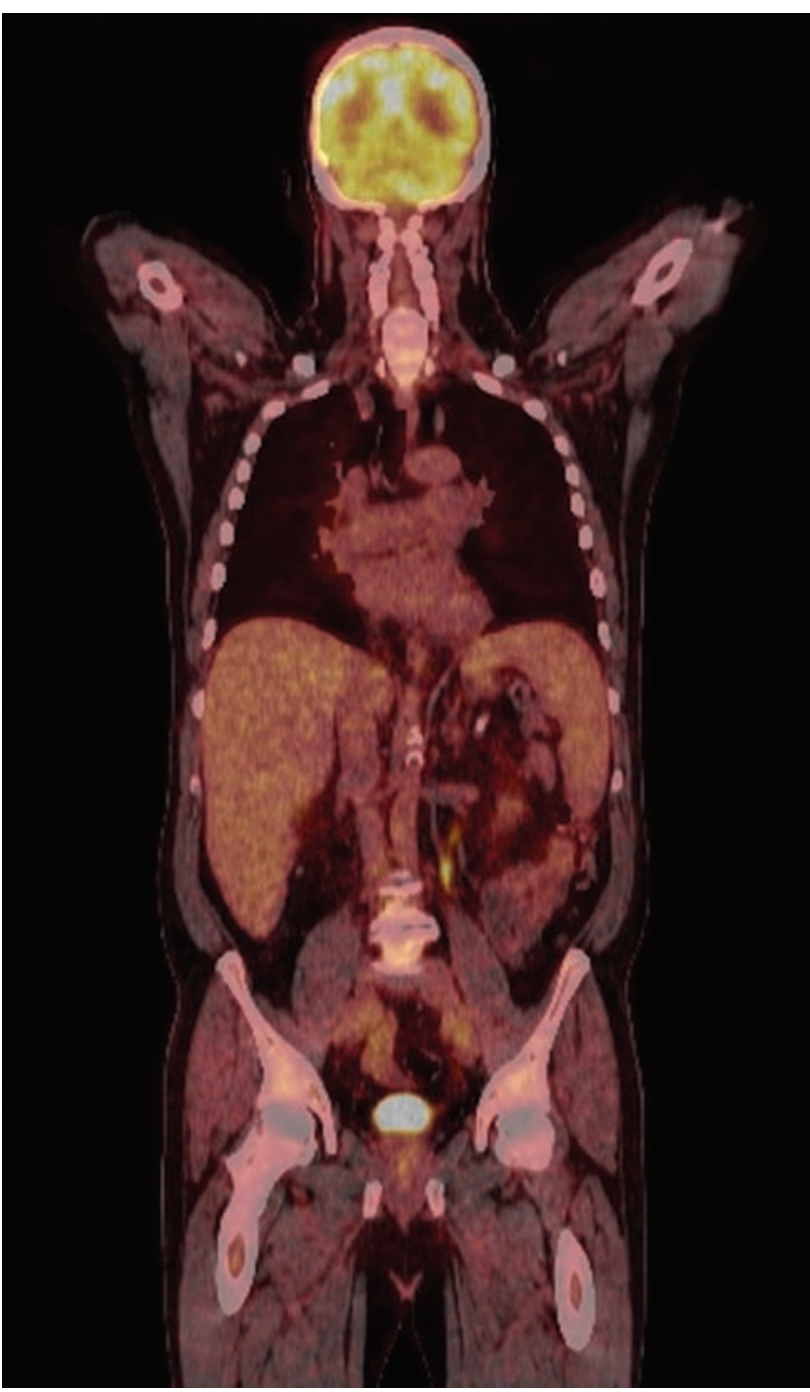

Fig 1. Positron emission tomography-computed tomography showing increased bone marrow uptake, hepato-splenomegaly and no evidence of systemic vasculitis.

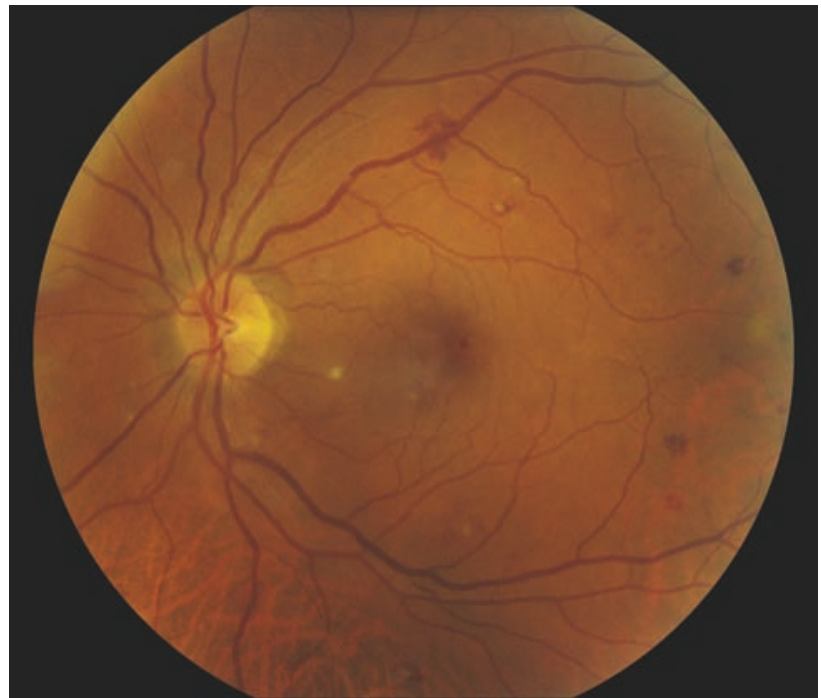

Fig 2. Fundoscopy showing Roth spots.

A diagnosis of native aortic valve IE was finally made, and the patient was managed by a specialist multidisciplinary IE team with cardiology, cardiothoracic surgery, clinical microbiology and antimicrobial pharmacy expertise. His antibiotics were rationalised to intravenous (IV) benzylpenicillin as the $S$ sinensis was very sensitive to penicillin (penicillin minimal inhibitory concentration = $0.008 \mathrm{mg} / \mathrm{L}$ ). Human immunodeficiency virus, hepatitis B and C serologies were negative. In view of the protracted illness and risk of mycotic aneurysm, magnetic resonance angiography of the head was requested but was negative. An orthopantomogram was unremarkable.

Two weeks later, the patient underwent an aortic valve replacement with no postoperative complications. Microscopy of valve tissue showed Gram-positive cocci, but culture did not yield any organisms. The patient required IV hydrocortisone in the peri-operative period given his chronic steroid exposure and was

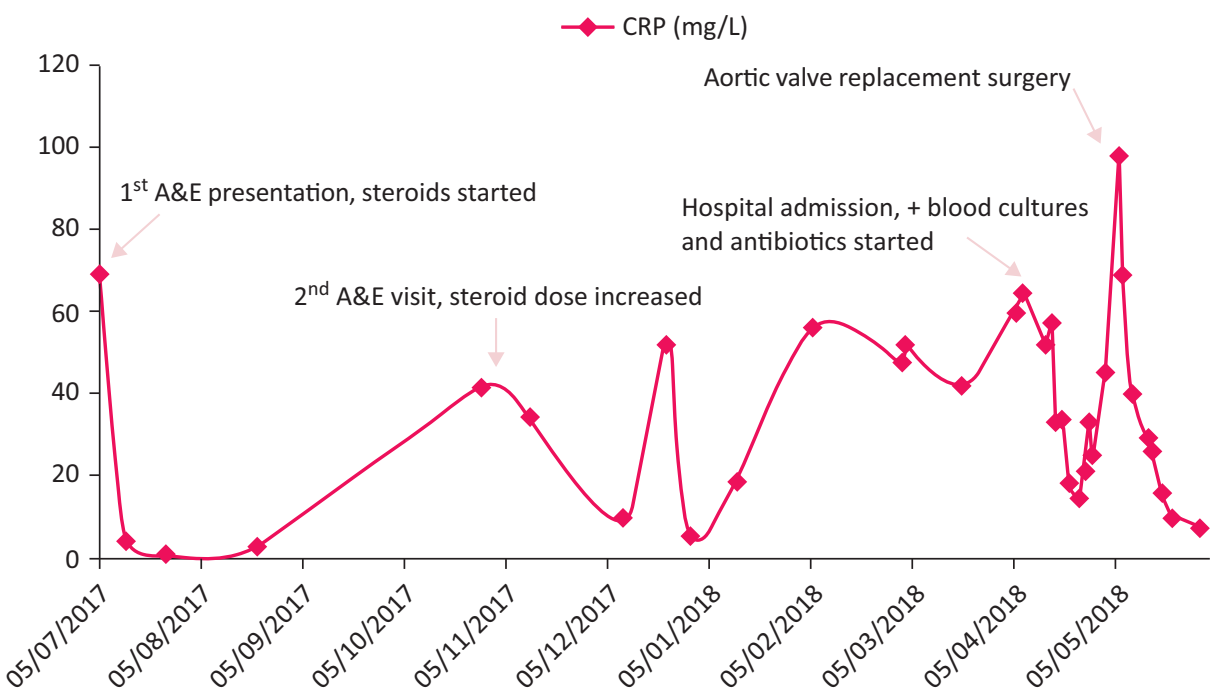

Fig 3. Patient's timeline and C-reactive protein values $(\mathrm{mg} / \mathrm{L})$. 
Fig 4. Transoesophageal echo demonstrating large vegetations on the aortic valve.

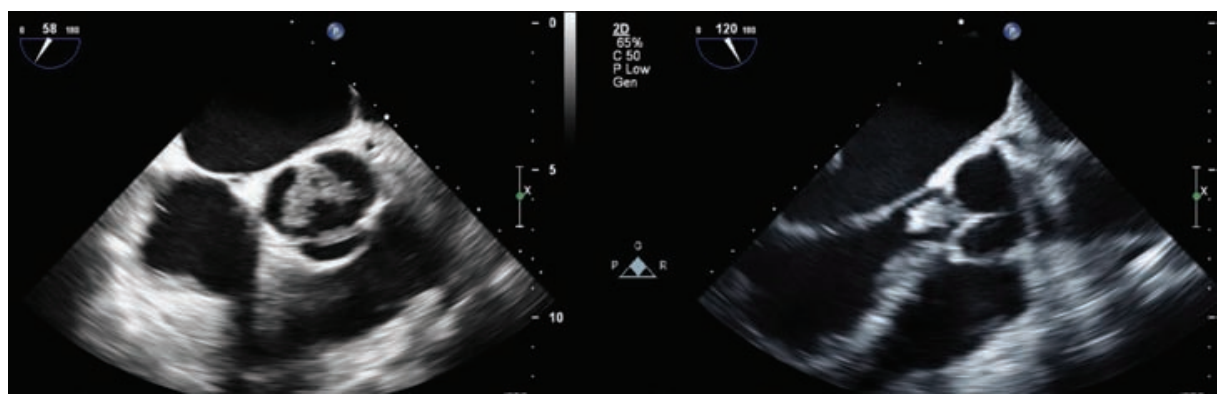

eventually weaned off corticosteroids. He completed 4 weeks of IV antibiotics postoperatively: 2 weeks of IV benzylpenicillin and 2 weeks IV ceftriaxone $2 \mathrm{~g}$ once daily via outpatient parenteral antibiotic therapy and reported full resolution of symptoms months later.

\section{Discussion}

S sinensis is a facultative anaerobic, Gram-positive coccus which forms alpha-haemolytic colonies on blood agar. ${ }^{1}$ It was first identified as a new species of viridans streptococci causing IE in Hong-Kong in 2002. ${ }^{1}$ It has subsequently been reported in a small number of case reports from France, ${ }^{2,3}$ Switzerland ${ }^{4}$ and Thailand, ${ }^{5}$ highlighting its importance as an emerging pathogen worldwide. S sinensis has been found as part of the oral flora in healthy individuals, ${ }^{6}$ similar to other viridans streptococci.

Infective endocarditis caused by viridans streptococci is commonly slow and insidious in presentation. The median interval between onset of symptoms and diagnosis is typically around 6 weeks, compared to this case where the diagnosis took 9 months. The mortality rate is $4-16 \%$, lower than with other pathogens (enterococci 15-25\%, Staphylococcus aureus $25-47 \%$, Pseudomonas aeruginosa and fungi $50 \%)^{7}$

We hypothesise that this patient's oral cavity became colonised with $S$ sinensis during his frequent trips to Asia and it is possible that he developed IE following a bacteraemic episode at the time of his dental procedure. This case illustrates the multisystem involvement in IE and the complex process of diagnosis. This patient's symptoms initially appeared quite typical for GCA and the use of corticosteroids masked the infection, leading to a 9-month delay in reaching the correct diagnosis. The case highlights the importance of considering alternative diagnoses, especially when there is incomplete resolution of symptoms and multiple attempts to wean steroids are unsuccessful.

\section{Learning points}

$>$ Infective endocarditis can be difficult to diagnose and is considered the great mimicker, leading to treatment delay.
> Taking blood cultures when the patient is not on antibiotics is critical when IE is suspected.

$>$ S sinensis is an emerging species of viridans streptococci associated with infective endocarditis worldwide.

> When a patient is not responding to treatment, consider alternative diagnoses.

\section{Consent}

Written consent was obtained from the patient for publication of this article.

\section{References}

1 Woo PCY, Tam DMW, Leung K-W et al. Streptococcus sinensis sp. nov., a novel species isolated from a patient with infective endocarditis. J Clin Microbiol 2002;40:805-10.

2 Faibis F, Mihaila L, Perna S et al. Streptococcus sinensis: an emerging agent of infective endocarditis. J Med Microbiol 2008;57:528-31.

3 Seta V, Teicher E, Fortineau N, Ladouceur M, Lambotte O. Endocardite infectieuse à Streptococcus sinensis. Med Mal Infect 2015;45:56-7.

4 Uçkay I, Rohner P, Bolivar I et al. Streptococcus sinensis endocarditis outside Hong Kong. Emerg Infect Dis 2007;13:1250-2.

5 Watt G, Pachirat O, Baggett HC et al. Infective endocarditis in Northeastern Thailand. Emerg Infect Dis 2014:20:473-6.

6 Woo PCY, Teng JLL, Tsang SNY, Tse CWS, Lau SKP, Yuen KY. The oral cavity as a natural reservoir for Streptococcus sinensis. Clin Microbiol Med 2001:14:1075-9.

7 Mylonakis E, Calderwood S. Infective endocarditis in adults. N Engl J Infect 2008;345:1318-30.

Address for correspondence: Dr Alberto San Francisco, Microbiology and Infection, Microbiology Department, Brighton and Sussex University Hospitals NHS Trust, Brighton, East Sussex, BN2 5BE, UK.

Email: albertosanfrancisco@nhs.net 